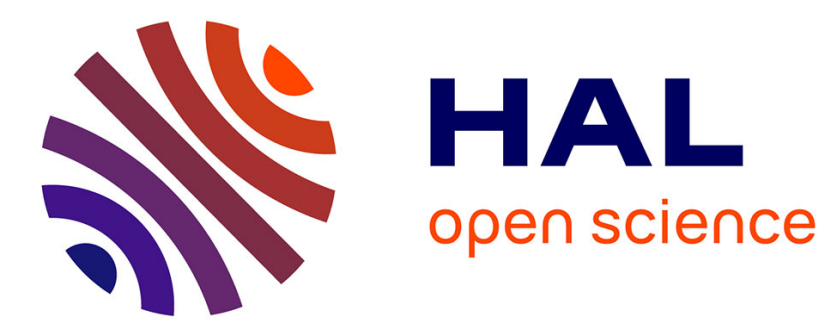

\title{
La première valeur propre de l'opérateur de Dirac sur les variétés kählériennes compactes
}

\author{
Andrei Moroianu
}

\section{To cite this version:}

Andrei Moroianu. La première valeur propre de l'opérateur de Dirac sur les variétés kählériennes compactes. Communications in Mathematical Physics, 1995, 169, pp.373-384. 10.1007/BF02099477 . hal-00125977

\section{HAL Id: hal-00125977 \\ https://hal.science/hal-00125977}

Submitted on 23 Jan 2007

HAL is a multi-disciplinary open access archive for the deposit and dissemination of scientific research documents, whether they are published or not. The documents may come from teaching and research institutions in France or abroad, or from public or private research centers.
L'archive ouverte pluridisciplinaire HAL, est destinée au dépôt et à la diffusion de documents scientifiques de niveau recherche, publiés ou non, émanant des établissements d'enseignement et de recherche français ou étrangers, des laboratoires publics ou privés. 


\title{
LA PREMIERE VALEUR PROPRE DE L'OPERATEUR DE DIRAC SUR LES VARIETES KAHLERIENNES COMPACTES
}

\author{
Andrei Moroianu
}

\section{Introduction}

En 1980, T.Friedrich ([Fr]) a montré à l'aide de la formule de Lichnerowicz et en utilisant une modification de la connexion de Levi-Civitá, que sur une variété riemannienne spinorielle compacte $\left(M^{n}, g\right)$, toute valeur propre $\lambda$ de l'opérateur de Dirac satisfait

$$
\lambda^{2} \geq \frac{n}{4(n-1)} \inf _{M} S
$$

où $S$ est la courbure scalaire de $M$. En 1984, O. Hijazi ([Hi1]) a montré que l'égalité ne peut être atteinte en (1) si $M$ est kählérienne. Le cas kählérien a été considéré par Kirchberg ([Ki1]) qui a montré que toute valeur propre $\lambda$ de l'opérateur de Dirac sur une variété kählérienne spinorielle compacte $\left(M^{2 m}, g\right)$ satisfait

$$
\lambda^{2} \geq \frac{m+1}{4 m} \inf _{M} S, \quad \text { si } m \text { est impair }
$$

et

$$
\lambda^{2} \geq \frac{m}{4(m-1)} \inf _{M} S, \quad \text { si } m \text { est pair }
$$

Pour simplifier, on appellera une variété-limite une variété kählérienne spinorielle compacte $\left(M^{2 m}, g, J\right)$ de dimension complexe impaire, pour laquelle l'égalité dans (2) est satisfaite.

Le cas d'égalité dans (3) a été analisé par Lichnerowicz [Li], qui a montré que le problème se réduit au cas de la dimension complexe impaire.

En 1988, Kirchberg ([Ki2]) classifie toutes les variétés-limites de dimension 6, et il trouve l'espace projectif complexe $\mathbf{C P}^{3}$ et la variété de drapeaux $F_{3}(1,2)$.

En [Hi2], Hijazi définit l'opérateur de twisteurs kählérien qui permet de démontrer l'inégalité (2) de manière naturelle et sans faire appel aux valeurs propres de la forme de Kähler. L'intérêt de cette approche est que le cas-limite de (2) se caractérise par l'existence d'un champ spinoriel parallèle pour une connexion modifiée. Plus précisement, il prouve

Théorème 1 ([Hi2]). Soit $M$ une variété-limite et $\Psi$ un spineur propre qui satisfait (2). Alors $M$ est un espace d'Einstein, $\Psi$ est un spineur-twisteur Kählérien et $\Psi=\Psi_{+}+\Psi_{-}$, avec $\Omega \cdot \Psi_{ \pm}= \pm(-1)^{\frac{m-1}{2}} i \Psi_{ \pm}$, où $\Psi_{+}$et $\Psi_{-}$sont les demi-spineurs associés à $\Psi$ dans la décomposition $\Sigma M=\Sigma^{+} M+\Sigma^{-} M$. 
Dans ce qui suit, on considérera que la métrique sur les variétés-limites est toujours normalisée de telle façon que $S=n(n+2)$.

Le but de ce papier est de montrer le résultat suivant

Théorème A. La seule variété-limite de dimension $8 l+2$ est $\mathbf{C} P^{4 l+1}$. Les variétés-limites de dimension $8 l+6$ sont exactement les espaces de twisteurs en sens généralisé [Sa] associés aux variétés quaternioniennes à courbure scalaire positive.

L'idée de la démonstration est d'utiliser Théorème 1 pour montrer que l'égalité dans (2) implique l'existence de spineurs de Killing sur un certain fibré en cercles $U M$ au-dessus de $M$. La classification due à C. Bär des variétés admettant des spineurs de Killing réels [Bä] permet de montrer que $U M$ admet une 3-structure de Sasaki régulière, et on remarque que $M$ est l'espace de twisteurs associé au quotient de $U M$ par les orbites de la 3-structure de Sasaki, en utilisant les resultats de Boyer, Galicki et Mann [BGM].

\section{Préliminaires}

Soit $\left(M^{2 m}, g, J\right)$ une variété kählérienne ; on appelle $\nabla$ la connexion de Levi-Civitá sur le fibré tangent $T M$, ainsi que son extension au fibré des formes extérieures, et au fibré des spineurs complexes, $\Sigma M$. L'opérateur de Dirac est défini par

$$
D=\sum_{i=1}^{n} e_{i} \cdot \nabla_{e_{i}}
$$

où $\left(e_{1}, \cdots, e_{n}\right)$ est une base locale orthonormée de $T M$. A partir de $J$, on définit $\widetilde{D}$, une autre "racine carrée du laplacien", par la formule (locale)

$$
\widetilde{D}=\sum_{i=1}^{n} J\left(e_{i}\right) \cdot \nabla_{e_{i}}=-\sum_{i=1}^{n} e_{i} \cdot \nabla_{J\left(e_{i}\right)}
$$

et on vérifie sans difficulté les relations

$$
\widetilde{D}^{2}=D^{2} \quad \text { et } \quad \widetilde{D} D+D \widetilde{D}=0 .
$$

On considère l'opérateur des twisteurs kählérien $P: \Sigma M \rightarrow T^{*} M \otimes \Sigma M$ défini par (cf. [Hi2])

$$
P_{X}(\Psi)=\nabla_{X} \Psi+\frac{1}{n+2} X \cdot D \Psi+\frac{1}{n+2} J(X) \cdot \widetilde{D} \Psi
$$

Définition. Un spineur $\Psi$ qui satisfait $P(\Psi) \equiv 0$ s'appelle un spineur-twisteur kählérien. 
L'action des $k$-formes sur $\Sigma M$ est donnée par

$$
\omega \cdot \Psi=\sum_{i_{1}<\cdots<i_{k}} \omega\left(e_{i_{1}}, \cdots, e_{i_{k}}\right) e_{i_{1}} \cdot \ldots \cdot e_{i_{k}} \cdot \Psi
$$

Modulo cette action, la forme de Kähler $\Omega$ (définie par $\Omega(X, Y)=g(X, J Y)$ ), satisfait

$$
\Omega=\frac{1}{2} \sum_{i=1}^{n} J\left(e_{i}\right) \cdot e_{i}=-\frac{1}{2} \sum_{i=1}^{n} e_{i} \cdot J\left(e_{i}\right) .
$$

L'action de $\Omega$ sur $\Sigma M$ donne une décomposition en somme directe (cf. [Ki1])

$$
\Sigma M=\oplus_{r=0}^{m} \Sigma_{r} M
$$

où $\Sigma_{r} M$ est le fibré propre de rang $C_{m}^{r}$ associé à la valeur propre $i \mu_{r}=i(m-2 r)$ de $\Omega$. Par rapport à cette décomposition, tout spineur $\Psi$ s'écrit d'une manière unique comme

$$
\Psi=\sum_{r=0}^{m} \Psi_{r}
$$

On aura besoin des formules élémentaires pour les submersions riemanniennes ([O'N1]). Pour toute submersion riemannienne $N \rightarrow M$, on définit les tenseurs $A$ et $T \operatorname{sur} N$ par

$$
\begin{aligned}
& A_{X} Y=\mathcal{V} \nabla_{\mathcal{H} \mathcal{X}} \mathcal{H} \mathcal{Y}+\mathcal{H} \nabla_{\mathcal{H} \mathcal{X}} \mathcal{V} \mathcal{Y} \\
& T_{X} Y=\mathcal{V} \nabla_{\mathcal{V} \mathcal{X}} \mathcal{H} \mathcal{Y}+\mathcal{H} \nabla_{\mathcal{V} \mathcal{X}} \mathcal{V} \mathcal{Y}
\end{aligned}
$$

où $\mathcal{H}$ et $\mathcal{V}$ sont la projection horizontale et verticale, respectivement.

Dans le cas où la fibre est de dimension 1 et totalement géodésique, les tenseurs $A$ et $T$ s'expriment par

$$
\begin{aligned}
& T=0 \quad, \quad A_{X^{*}} V=\nabla_{X^{*}} V=\nabla_{V} X^{*}, \\
& A_{X^{*}} Y^{*}=-A_{Y^{*}} X^{*}=\frac{1}{2} \mathcal{V}\left(\left[X^{*}, Y^{*}\right]\right),
\end{aligned}
$$

où $X^{*}$ est le relèvement horizontal de $X \in T M$ en un point $y \in N$, et ici, ainsi que dans tout ce qui suit, $V$ est le vecteur unitaire en $y$ tel que $\left\{X_{1}^{*}, \cdots, X_{n}^{*}, V\right\}$ est une base orientée de $T_{y} N$ pour toute base orientée $\left\{X_{1}, \cdots, X_{n}\right\}$ de $T_{\pi(y)} M$.

On conclut cette section introductive avec les définitions suivantes

Définition. Un champ vectoriel $X$ sur une variété riemannienne $(M, g)$ s'appelle une structure de Sasaki si les conditions suivantes sont vérifiées

1. $X$ est un champ vectoriel de Killing de longueur constante 1 ;

2. Le tenseur $\varphi$ de type $(1,1)$ défini par $\varphi=-\nabla X$ est une structure presque complexe sur la distribution orthogonale à $X\left(\varphi^{2}=-1\right.$ et $\varphi=-\varphi^{*}$ sur $\left.X^{\perp}\right)$; 
3. $\left(\nabla_{V} \varphi\right) W=g(V, W) X-g(X, W) V, \quad \forall U, V$.

Définition. Un triplet $\left(X_{1}, X_{2}, X_{3}\right)$ s'appelle une 3-structure de Sasaki sur M si les conditions suivantes sont vérifiées

1. Le vecteur $X_{i}$ définit une structure de Sasaki pour chaque $i \in\{1,2,3\}$;

2. Le repère $\left(X_{1}, X_{2}, X_{3}\right)$ est orthonormé;

3. Pour toute permutation $(i, j, k)$ de $(1,2,3)$ de signature $\delta$ on a $\nabla_{X_{i}} X_{j}=$ $(-1)^{\delta} X_{k}$;

4. Sur la distribution $\mathcal{H}$, orthogonale à $\left(X_{1}, X_{2}, X_{3}\right)$, les tenseurs $\varphi_{i}=-\nabla X_{i}$ satisfont $\varphi_{i} \varphi_{j}=(-1)^{\delta} \varphi_{k}$ pour toute permutation $(i, j, k)$ de $(1,2,3)$ de signature $\delta$.

\section{La construction de $U M$}

Cette section est dédiée au résultat suivant

Proposition 1. Pour toute variété-limite $\left(M^{2 m}, g, J\right)$, il existe une variété riemannienne spinorielle $U M$ de dimension $2 m+1$ et une submersion riemannienne $\pi: U M \rightarrow M$ dont les tenseurs fondamentaux (cf. [O'N1]) satisfont

$$
T=0 \quad \text { et } \quad A_{X^{*}} V=J(X)^{*} .
$$

Preuve. Toute variété-limite est une variété d'Einstein-Kähler, donc la classe de cohomologie de la forme de Kähler de $M$ est un multiple réel de la première classe de Chern de $M:[\Omega]=(2 \pi n / S) c_{1}(M)$. Soit $r$ le plus grand entier positif tel que $c_{1}(M) / r$ soit encore une classe entière. Considérons le fibré en droites complexes $P M$ sur $M$, dont la première classe de Chern, $c_{1}(P M \rightarrow M)$, est égale à $c_{1}(M) / r$. On choisit une métrique hermitienne arbitraire $h$ sur $P M$ et on considère $U M$, le fibré principal associé, de groupe structural $U(1)=S^{1}$. Soit $\sigma$ l'action (libre) de $S^{1}$ sur $U M$. On considère une connexion sur $U M$ de forme de connexion $\alpha$, qui induit une connexion métrique sur $P M$. Soit $F$ la forme de courbure de $\alpha$ sur $U M$. La connexion sur $U M$ induit une famille de métriques riemanniennes sur $U M$ qui font de $\pi$ une submersion riemannienne : il suffit de définir $g_{U M}^{c}(X, Y)=g_{M}\left(\pi_{*}(X), \pi_{*}(Y)\right)-c^{2} \alpha(X) \alpha(Y) \quad(c>0)$, via l'identification de $\mathcal{L}\left(\mathcal{S}^{\infty}\right)$ avec $i \mathbf{R}$ qui fait correspondre $\frac{\partial}{\partial t} \grave{a} i$. La submersion riemannienne ainsi obtenue est à fibres totalement géodésiques. D'une manière évidente on a

$$
\left[\pi^{*} \Omega\right]=\frac{2 \pi n}{S} c_{1}(M)=\frac{2 \pi n r}{S} c_{1}(P M \rightarrow M)=\frac{i n r}{S}[F] .
$$

Après une éventuelle modification de la connexion sur $U M$, on a donc les relations

$$
\pi^{*} \Omega=\frac{i n r}{S} F
$$


et

$$
\begin{aligned}
F\left(X^{*}, Y^{*}\right) & =d \alpha\left(X^{*}, Y^{*}\right) \\
& =-\frac{1}{2} \alpha\left(\left[X^{*}, Y^{*}\right]\right) \\
& =\frac{1}{2 c i} g_{U M}^{c}\left(\left[X^{*}, Y^{*}\right], V\right) \quad\left(V=(1 / c) \sigma_{*}\left(\frac{\partial}{\partial t}\right)\right),
\end{aligned}
$$

donc, finalement,

$$
\begin{aligned}
\pi^{*} \Omega\left(X^{*}, Y^{*}\right) & =\frac{n r}{2 c S} g_{U M}^{c}\left(\left[X^{*}, Y^{*}\right], V\right) \\
& =\frac{n r}{c S} A_{X} Y \\
& =\frac{n r}{c S} g_{U M}^{c}\left(\nabla_{X^{*}} Y^{*}, V\right) \\
& =-\frac{n r}{c S} g_{U M}^{c}\left(Y^{*}, \nabla_{X^{*}} V\right) \\
& =-\frac{n r}{c S} g_{M}\left(Y, \pi\left(A_{X^{*}} V\right)\right)
\end{aligned}
$$

Pour $c=n r / S$, on obtient

$$
\Omega(X, Y)=-g_{M}\left(Y, \pi\left(A_{X^{*}} V\right)\right)
$$

qui donne

$$
A_{X^{*}} V=J(X)^{*} .
$$

En fait, la variété $U M$ est une racine maximale du fibré en droites complexes canonique sur $M$. On conclut la démonstration de la proposition avec le lemme suivant.

Lemme . Pour toute submersion riemannienne $p: E \rightarrow M$ à fibres de dimension 1 et pour toute structure spinorielle sur $M$, il existe une structure spinorielle naturelle sur $E$ provenant de celle sur $M$.

Preuve. Soient $P_{\mathrm{SO}(n)}(M)$ et $P_{\mathrm{Spin}(n)}(M)$ le fibré des repères orthonormés orientés et la structure spinorielle sur $M$ respectivement, et $P_{\mathrm{SO}_{(n+1)}}(M)$ et $P_{\operatorname{Spin}(n+1)}(M)$ les fibrés obtenus par l'agrandissement des groupes structuraux. On considère aussi le fibré des repères orthonormés orientés sur $E, P_{\mathrm{SO}_{(n+1)}}(E)$. On définit

$$
g: P_{\mathrm{SO}(n+1)}(E) \longmapsto P_{\mathrm{SO}_{(n+1)}}(M)
$$

par

$$
g\left(\left(u^{*}, V\right) \cdot A\right)=[u, A]
$$


car tout repère sur $E$ s'écrit $\left(u^{*}, V\right) \cdot A$, où $u^{*}$ est le relèvement d'un repère sur $M$ et $A \in \mathrm{SO}(n+1)$. On vérifie facilement que $g$ est bien défini. On a donc un diagramme commutatif

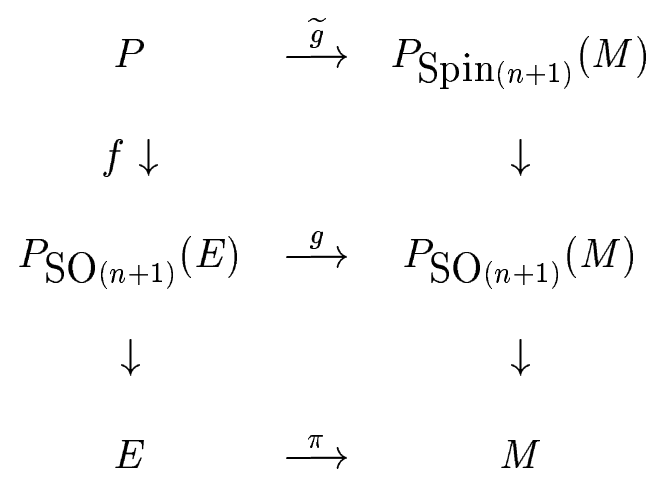

où $P$ est le pull-back de $P_{\operatorname{Spin}(n+1)}(M)$ par $\pi$ et $f$ est défini par

$$
f([\widetilde{u}, \alpha], e)=\left(\left(u^{*}, V\right)_{e} \cdot \theta(\alpha)\right) .
$$

Ici $\widetilde{u} \in P_{\operatorname{Spin}(n)}(M), \alpha \in \operatorname{Spin}(n+1), u^{*}$ est le relèvement dans $e$ de la projection de $\widetilde{u}$ dans $P_{\mathrm{SO}(n)}(M)$ et $\theta$ est la projection de $\operatorname{Spin}(n+1)$ sur $\mathrm{SO}(n+1)$. La vérification du fait que $f$ est bien définie et représente une structure spinorielle sur $E$ est triviale. Ceci achève la démonstration du lemme et de la proposition.

Q.E.D.

Pour $E=U M$, on appelle $\nabla$ la connexion de Levi-Civitá sur $U M$, ainsi que la connexion induite sur le fibré des spineurs sur $U M$.

\section{Spineurs projetables}

Les résultats de cette section sont valables pour toute submersion riemannienne $E \rightarrow M$ à fibres totalement géodésiques de dimension 1 sur une variété $M$ de dimension paire. Soit, pour $n=2 m, \Sigma_{n}$ - la représentation irréductible de $\mathrm{Cl}_{n}$. Par rapport à l'action de $\operatorname{Spin}(n), \Sigma_{n}$ se décompose en

$$
\Sigma_{n}=\Sigma_{n}^{+} \oplus \Sigma_{n}^{-} .
$$

Si par rapport à cette décomposition, $\psi$ s'écrit $\psi=\psi_{+}+\psi_{-}$, on note $\bar{\psi}=\psi_{+}-\psi_{-}$.

L'algèbre $\mathbf{C l}_{n+1}$ a deux représentations irréductibles, $\Sigma_{n+1}^{ \pm}$, dont la somme directe est la représentation spinorielle $\Sigma_{n+1}$. Les sous-espaces $\Sigma_{n+1}^{ \pm}$de $\Sigma_{n+1}$ sont les sous-espaces propres de la multiplication par $\omega=i^{m} e_{1} \cdots e_{n+1}$ avec la valeur propre $\pm i$. On identifie $\Sigma_{n+1}^{ \pm}$avec $\Sigma_{n}$ par les actions $\rho^{ \pm}$de $\mathbf{C l}_{n+1}$ sur $\Sigma_{n}$ suivantes

$$
\rho^{ \pm}\left(e_{k}\right) \cdot \psi= \begin{cases}e_{k} \cdot \psi & \text { si } k \leq n \\ \pm i \cdot \bar{\psi} & \text { si } k=n+1\end{cases}
$$


Dans ce qui suit, on identifie $\Sigma_{n+1}^{+}$avec $\Sigma_{n}$ par l'intermédiaire de $\rho^{+}$. En utilisant les notations de la section précédente, on définit $h: \Sigma^{+} E \rightarrow \Sigma M$ par la formule

$$
h([\widetilde{v}, \psi])=[\widetilde{g}(\widetilde{v}), \psi],
$$

et on vérifie facilement qu'il est bien défini. Evidement, $\Sigma^{+} E=\pi^{*}(\Sigma M)$.

Définition. On appelle spineur projetable tout champ spinoriel $\Psi$ sur $E$ qui satisfait $h\left(\Psi_{e}\right)=h\left(\Psi_{f}\right)$ pour tous e et $f$ tels que $\pi(e)=\pi(f)$.

Tout spineur projetable $\Phi$ sur $E$ induit d'une manière évidente un spineur $h(\Phi)$ sur $M$. Réciproquement, tout spineur $\Psi$ sur $M$ induit un unique spineur projetable $\widetilde{\Psi}$ sur $E$ par la formule $\widetilde{\Psi}=\pi^{*} \Psi$. Dans un certain sens, les spineurs projetables sont les spineurs sur $E$ "constants" le long des fibres de la fibration $E \rightarrow M$.

Si $\widetilde{\Phi}$ est projetable sur $\Phi$, alors $X^{*} \cdot \widetilde{\Phi}$ est projetable sur $X \cdot \Phi$ et $V \cdot \widetilde{\Phi}$ est projetable sur $i \bar{\Phi}$.

Proposition 2. Soit $\widetilde{\Phi}$ un spineur sur E, projetable sur $\Phi$. Alors $\widetilde{\nabla}_{X^{*}} \widetilde{\Phi}$ est projetable sur

$$
\nabla_{X} \Phi-\frac{1}{2} i \pi_{*}\left(A_{X^{*}} V\right) \cdot \bar{\Phi}
$$

et $\widetilde{\nabla}_{V} \widetilde{\Phi}$ est projetable sur

$$
-\frac{1}{4} \sum_{j=1}^{n} \pi_{*}\left(A_{X_{j}^{*}} V\right) \cdot X_{j} \cdot \Phi .
$$

Preuve. Soit $s=\left(X_{1}, \cdots, X_{n}\right)$ une section locale de $P_{\mathrm{SO}_{(n)}}(M)$ qui induit une section $\left[s, I_{n+1}\right]$ de $P_{\mathrm{SO}(n+1)}(M)$ et une section $s_{0}$ de $P_{\operatorname{Spin}(n+1)}(M)$. A son tour, $s_{0}$ induit une section $\widetilde{s}(e)=\left(s_{0}(\pi(e)), e\right)$ de la structure spinorielle $P_{\operatorname{Spin}(n+1)}(E)$. Si le spineur $\Phi$ s'écrit localement $\Phi=[s, \phi]$, où $\phi: U \subset M \rightarrow \Sigma_{n}$ alors $\widetilde{\Phi}=[\widetilde{s}, \psi]$, où $\psi=\phi \circ \pi$, et on a

$$
\begin{aligned}
\widetilde{\nabla}_{X^{*}} \widetilde{\Phi}= & \frac{1}{2} \sum_{j<k} X_{j}^{*} \cdot X_{k}^{*}\left\langle\widetilde{\nabla}_{X^{*}} X_{j}^{*}, X_{k}^{*}\right\rangle \cdot \widetilde{\Phi}+\left[\widetilde{s}, X^{*}(\psi)\right]+ \\
& +\frac{1}{2} \sum X_{j}^{*} \cdot V\left\langle\widetilde{\nabla}_{X^{*}} X_{j}^{*}, V\right\rangle \cdot \widetilde{\Phi} \\
= & \frac{1}{2} \sum_{j<k} X_{j}^{*} \cdot X_{k}^{*}\left\langle\widetilde{\nabla}_{X^{*}} X_{j}^{*}, X_{k}^{*}\right\rangle \cdot \widetilde{\Phi}+[\widetilde{s}, X(\phi)]- \\
& -\frac{1}{2} A_{X^{*}} V \cdot V \cdot \widetilde{\Phi}
\end{aligned}
$$


qui est projetable sur

$$
\begin{gathered}
\frac{1}{2} \sum_{j<k} X_{j} \cdot X_{k}\left\langle\nabla_{X} X_{j}, X_{k}\right\rangle \cdot \Phi+[s, X(\phi)]-\frac{1}{2} \pi_{*}\left(A_{X^{*}} V\right) \cdot V \cdot \Phi= \\
=\nabla_{X} \Phi-\frac{1}{2} i \pi_{*}\left(A_{X^{*}} V\right) \cdot \bar{\Phi} .
\end{gathered}
$$

La deuxième partie de la proposition se démontre de la même manière :

$$
\begin{aligned}
\widetilde{\nabla}_{V} \widetilde{\Phi}= & \frac{1}{2} \sum_{j<k} X_{j}^{*} \cdot X_{k}^{*}\left\langle\widetilde{\nabla}_{V} X_{j}^{*}, X_{k}^{*}\right\rangle \cdot \widetilde{\Phi}+[\widetilde{s}, V(\psi)]+ \\
& +\frac{1}{2} \sum X_{j}^{*} \cdot V\left\langle\widetilde{\nabla}_{V} X_{j}^{*}, V\right\rangle \cdot \widetilde{\Phi} \\
= & \frac{1}{2} \sum_{j<k} X_{j}^{*} \cdot X_{k}^{*}\left\langle\widetilde{\nabla}_{V} X_{j}^{*}, X_{k}^{*}\right\rangle \cdot \widetilde{\Phi} \\
= & \frac{1}{4} \sum_{j, k} X_{j}^{*} \cdot X_{k}^{*}\left\langle\widetilde{\nabla}_{V} X_{j}^{*}, X_{k}^{*}\right\rangle \cdot \widetilde{\Phi} \\
= & \frac{1}{4} \sum_{j} X_{j}^{*} \cdot \widetilde{\nabla}_{V} X_{j}^{*} \cdot \widetilde{\Phi} \\
= & \frac{1}{4} \sum_{j} X_{j}^{*} \cdot \pi_{*}\left(A_{X_{j}^{*}} V\right) \cdot \widetilde{\Phi}
\end{aligned}
$$

qui est projetable sur $-\frac{1}{4} \sum_{j=1}^{n} \pi_{*}\left(A_{X_{j}^{*}} V\right) \cdot X_{j} \cdot \Phi$.

Q.E.D.

Corollaire. Si $D^{E}$ et $D$ sont les opérateurs de Dirac sur $E$ et $M$ respectivement, alors pour tout spineur $\widetilde{\Phi}$ projetable sur $\Phi, D^{E} \widetilde{\Phi}$ est projetable sur $D \Phi-\frac{1}{4} i \sum_{j=1}^{n} X_{j} \pi_{*}\left(A_{X_{j}^{*}} V\right) \cdot \bar{\Phi}$. En particulier, pour $E=U M$ on a

$$
h\left(D^{U M} \widetilde{\Phi}\right)=D \Phi+\frac{1}{2} i \Omega \cdot \bar{\Phi} .
$$

\section{$5 \quad$ Spineurs de Killing}

Dans cette section $E$ sera une variété riemannienne spinorielle de dimension impaire $2 m+1$ avec la connexion de Levi-Civitá $\widetilde{\nabla}$. Les résultats qu'on obtient seront utilisés dans les sections suivantes dans le cas où $E$ est le fibré $U M$ sur $M$ construit ci-dessus.

Soit $C E$ le cône sur $E$, c.à.d. $C E=E \times \mathbf{R}_{+}^{*}$ avec la métrique $\bar{g}=r^{2} g_{E}+d r^{2}$. Alors la dérivée covariante $\bar{\nabla}$ de la connexion de Levi-Civitá de $\bar{g}$ satisfait les formules des produits tordus ([O'N2], p.206)

$$
\bar{\nabla}_{\frac{\partial}{\partial r}} \frac{\partial}{\partial r}=0
$$




$$
\begin{gathered}
\bar{\nabla}_{\frac{\partial}{\partial r}} X=\bar{\nabla}_{X} \frac{\partial}{\partial r}=\frac{1}{r} X, \\
\bar{\nabla}_{X} Y=\widetilde{\nabla}_{X} Y-r\langle X, Y\rangle_{E} \frac{\partial}{\partial r},
\end{gathered}
$$

pour tous champs de vecteurs $\mathrm{X}$ et $\mathrm{Y}$ sur $E$, identifiés aussi avec leurs prolongements canoniques à $C E$.

On identifie $\Sigma_{2 m+2}^{+}$avec $\Sigma_{2 m+1}$ par l'isomorphisme de $\mathbf{C l}_{2 m+2}^{+}$sur $\mathbf{C l}_{2 m+1}$ donné par $e_{i} \cdot e_{2 m+2} \mapsto e_{i}$. On applique, mutatis mutandis, les mêmes raisonnements des deux sections précédentes, et on trouve (en utilisant (6), (7), (8))

Proposition 3. Soit $\widetilde{\Phi}$ un spineur sur $C E$, projetable sur $\Phi$. Alors $\bar{\nabla}_{X} \widetilde{\Phi}$ est projetable sur

$$
\widetilde{\nabla}_{X} \Phi-\frac{1}{2} X \cdot \Phi
$$

et $\bar{\nabla}_{\frac{\partial}{\partial t}} \widetilde{\Phi}=0$.

Donc si $E$ admet un spineur de Killing de constante $-\frac{1}{2}, C E$ admet un spineur parallèle. Ceci est une manière plus simple de classifier les variétés simplement connexes admettant des spineurs de Killing, sans passer par les formes de connexion et les groupes d'holonomie, comme dans le papier initial de Bär ([Bä]).

Remarque. Si $E$ admet un spineur de Killing de constante $\frac{1}{2}$, alors $-C E$ admet un spineur parallèle, où $-C E$ est $C E$ avec l'orientation opposée. Ceci résulte tout simplement du fait que si on change l'orientation de $C E$, alors pour tout spineur $\widetilde{\Phi}$ sur $C E$ projetable sur $\Phi$, le spineur qui sera projetable sur $X \cdot \Phi$ est $X \cdot\left(-\frac{\partial}{\partial t}\right) \cdot \widetilde{\Phi}$ et non pas $X \cdot \frac{\partial}{\partial t} \cdot \widetilde{\Phi}$.

\section{Démonstration du théorème principal}

Soit maintenant $M$ une variété-limite de dimension $n=2 m, m$ impair, et $U M \rightarrow M$ la submersion riemannienne construite dans les sections précédentes. Supposons que sur $M$ il existe un spineur $\Psi$ qui satisfait $D \Psi=\lambda \Psi$, avec

$$
\lambda^{2}=\frac{n+2}{4 n} \inf _{M} S
$$

Le théorème 1 montre que $S$ est constant, et que

$$
\nabla_{X} \Psi+\frac{1}{n+2} X \cdot D \Psi+\frac{1}{n+2} J(X) \cdot \widetilde{D} \Psi=0
$$

On choisit un repère local orthonormé $\left(X_{1}, \cdots, X_{n}\right)$. On multiplie (9), pour $X=$ $X_{i}$, par $J\left(X_{i}\right)$, on somme sur $i$, et on obtient

$$
\widetilde{D} \Psi=-\Omega \cdot D \Psi=-\lambda \Omega \cdot \Psi .
$$


De la deuxième partie du théorème 1 on tire

$$
\widetilde{D} \Psi=-i \varepsilon \lambda \bar{\Psi}
$$

où $\varepsilon=(-1)^{\frac{m-1}{2}}$, donc on a

$$
\nabla_{X} \Psi+\frac{\lambda}{n+2} X \cdot \Psi-\frac{i \varepsilon \lambda}{n+2} J(X) \cdot \bar{\Psi}=0 .
$$

On a supposé au début que la métrique sur $M$ est renormalisée de telle manière que $S=n(n+2)$, donc $\lambda^{2}=\left(\frac{n+2}{2}\right)^{2}$, ce qui donne $\lambda= \pm \frac{n+2}{2}$. Du fait que $n$ est pair, le spectre de $D$ est symétrique par rapport à 0 , donc on peut supposer $\lambda=\frac{\varepsilon(n+2)}{2}$, où $\varepsilon=(-1)^{\frac{m-1}{2}}$. Alors (12) donne

$$
\nabla_{X} \Psi+\frac{\varepsilon}{2} X \cdot \Psi-\frac{i}{2} J(X) \cdot \bar{\Psi}=0 .
$$

Soit $\widetilde{\Psi}$ le spineur projetable induit par $\Psi$ sur $U M$. Par la proposition $2, \widetilde{\nabla}_{X^{*}} \widetilde{\Psi}$ est projetable sur $\nabla_{X} \Psi-\frac{1}{2} i \pi_{*}\left(A_{X^{*}} V\right) \cdot \bar{\Psi}$ et par la proposition $1, \pi_{*}\left(A_{X^{*}} V\right)=J(X)$. Ainsi, $\widetilde{\nabla}_{X^{*}} \widetilde{\Psi}$ est projetable sur $\nabla_{X} \Psi-\frac{1}{2} i J(X) \cdot \bar{\Psi}$. De plus, $\frac{\varepsilon}{2} X^{*} \cdot \widetilde{\Psi}$ est projetable sur $\frac{\varepsilon}{2} X \cdot \Psi$, donc (13) montre que

$$
\widetilde{\nabla}_{X^{*}} \widetilde{\Psi}+\frac{\varepsilon}{2} X^{*} \cdot \widetilde{\Psi}=0 \quad \forall X \in T M .
$$

D'autre part, de la deuxième partie de la proposition 2 on tire

$$
\begin{aligned}
h\left(\widetilde{\nabla}_{V} \widetilde{\Psi}\right) & =-\frac{1}{4} \sum_{j=1}^{n} \pi_{*}\left(A_{X_{j}^{*}} V\right) \cdot X_{j}^{*} \cdot \Psi \\
& =-\frac{1}{2} \Omega \cdot \Psi \\
& =-\frac{i \varepsilon}{2} \bar{\Psi} \\
& =h\left(-\frac{\varepsilon}{2} V \cdot \widetilde{\Psi}\right) .
\end{aligned}
$$

Donc $\widetilde{\Psi}$ est un spineur de Killing de $\Sigma^{+} U M$ de constante $-\frac{\varepsilon}{2}$. De plus, $\widetilde{\Psi}$ est un spineur projetable, donc par la proposition 2

$$
\begin{aligned}
h\left(-\frac{\varepsilon}{2} V \cdot \widetilde{\Psi}\right) & =h\left(\widetilde{\nabla}_{V} \widetilde{\Psi}\right) \\
& =-\frac{1}{4} \sum_{j=1}^{n} \pi_{*}\left(A_{X_{j}^{*}} V\right) \cdot X_{j} \cdot \Psi \\
& =-\frac{1}{4} \sum_{j=1}^{n} J\left(X_{j}\right) \cdot X_{j} \cdot \Psi \\
& =-\frac{1}{2} \Omega \cdot \Psi \\
& =-\frac{1}{2} h(\widetilde{\Omega} \cdot \widetilde{\Psi}),
\end{aligned}
$$


où $\widetilde{\Omega}=\frac{1}{2} \sum_{j=1}^{n} J\left(X_{j}^{*}\right) \cdot X_{j}^{*}$. Par conséquent,

$$
\varepsilon V \cdot \widetilde{\Psi}=\widetilde{\Omega} \cdot \widetilde{\Psi}
$$

Comme $M$ est une variété d'Einstein-Kähler de constante d'Einstein positive, le théorème de Kobayashi ([Ko]) montre que $M$ est simplement connexe. En considérant la suite exacte d'homotopie et la suite exacte de Thom-Gysin du fibré $\left(U M, \pi, M, S^{1}\right)$, on obtient que $U M$ est lui aussi simplement connexe (cf. $[\mathrm{M}-\mathrm{S}])$. La classification de Bär montre alors que le groupe d'holonomie du cône $C M$ sur $U M$ est un des groupes du tableau suivant

\begin{tabular}{|c||c|}
\hline$n=$ dimension de $M$ & $\operatorname{Hol}(C M)$ \\
\hline$n$ arbitraire & 0 \\
\hline$n+2=2 k$ & $\operatorname{SU}(k)$ \\
\hline$n+2=4 k$ & $\operatorname{Sp}(k)$ \\
\hline$n+2=8$ & $\operatorname{Spin}(7)$ \\
\hline$n+2=7$ & $G_{2}$ \\
\hline
\end{tabular}

Pour $n=6$, le cas $\operatorname{Hol}(C M)=\operatorname{Spin}(7)$ est impossible car $V$ définit une structure de Sasaki sur $U M$, donc $C M$ est kählérienne et Ricci-plate ce qui montre que le groupe d'holonomie de $C M$ est inclus dans $\mathrm{SU}(4) \subset \operatorname{Spin}(7)$ (strictement) (cf. [Bä], pp.14).

On montre à présent que le cas $\operatorname{Hol}(C M)=\mathrm{SU}(2 k)$ est, lui aussi, impossible. Considérons le relèvement parallèle $\Phi$ de $\widetilde{\Psi}$ dans $C M$, où l'orientation de $C M$ serait désormais $-\varepsilon$. La forme de Kähler de $C M$ est $\hat{\Omega}=\Omega+\widetilde{J}(V) \cdot V=\widetilde{\Omega}-\frac{\partial}{\partial t} \cdot V=$ $\widetilde{\Omega}+V \cdot \frac{\partial}{\partial t}$ (cf. [Bä], pp.13). De la section 5 on sait que $V \cdot\left(-\varepsilon \frac{\partial}{\partial t}\right) \cdot \Phi$ est projetable sur $V \cdot \widetilde{\Psi}$, donc $\hat{\Omega} \cdot \Phi$ est projetable sur $\widetilde{\Omega} \cdot \widetilde{\Psi}-\varepsilon V \cdot \widetilde{\Psi}$ qui est nul à cause de (14). Sur $C M$ on a donc trouvé un spineur parallèle qui se trouve dans le noyau de $\hat{\Omega}, \Sigma_{k} C M$. D'autre part, un raisonnement purement algébrique montre que les deux spineurs parallèles se trouvent respectivement dans $\Sigma_{0} C M$ et $\Sigma_{2 k} C M$, une contradiction. Les seuls cas qui restent sont par conséquent $\operatorname{Hol}(C M)=\operatorname{Sp}(k)$ et $\operatorname{Hol}(C M)=0$. Dans chacun de ces deux cas, $U M$ admet une 3-structure de Sasaki. Pour montrer sa régularité, il suffit de remarquer que, si $(V, X, Y)$ définit la 3-structure de Sasaki sur $U M$, alors la projection de $X$ et $Y$ sur $M$ est une distribution intégrable et régulière de dimension 2 sur $M$, dont les orbites sont des sphères $S^{2}$ (cf. [He]). Un raisonnement algébrique, montre que dans le cas $n=8 l+2$, il n'y a pas de spineur parallèle dans $\Sigma_{k} C M$, sauf si $C M=\mathbf{R}^{n+2}$, ce qui implique $M=\mathrm{CP}^{4 l+1}$. Finalement, le quotient de $U M$ par la 3-structure de Sasaki est une variété quaternionienne de courbure scalaire positive, et $M$ est l'espace des twisteurs associé à cette variété quaternionienne, car il est le quotient de $U M$ par une des structures de Sasaki (cf. [BGM]). 
Réciproquement, pour $n=8 l+6=4 k-2$, si $V^{n-2}$ est une variété quaternionienne de courbure scalaire positive, il existe un fibre, $N^{n+1}$, au-dessus de $V$, qui admet une 3-structure de Sasaki régulière. Soit $M$ le quotient de $N$ par l'orbite d'un des 3 vecteurs de Killing. Alors $M$ est une variété kählérienne spinorielle, étant l'espace des twisteurs de $V$. D'autre part, $N$ est une variété d'Einstein de courbure scalaire $n(n+1)$, car il admet une 3-structure de Sasaki. Alors les formules de [O'N1], pp.465 montrent que $M$ est une variété d'Einstein avec la courbure scalaire $S=n(n+2)$. Le cône sur $N, C N$, est hyperkählérien, donc il existe un spineur parallèle $\bar{\Psi}$ dans $\Sigma_{k} C N$, projetable sur un spineur $\widetilde{\Psi}$ sur $N$, qui par le paragraphe ci-dessus est à son tour projetable sur un spineur $\Psi$ sur $M$ qui satisfait (13). En plus, comme $\widetilde{\Psi}$ satisfait (14), on obtient

$$
\Omega \cdot \Psi=-i \bar{\Psi}
$$

donc $\Psi=\Psi_{+}+\Psi_{-}$, avec $\Omega \cdot \Psi_{ \pm}=\mp i \Psi_{ \pm}$. De (13) et (15) on tire

$$
D \Psi+\frac{n}{2} \Psi+\Psi=0,
$$

qui montre que $\Psi$ est un spineur propre avec $\lambda=\frac{-(n+2)}{2}$, i.e. $M$ est une variétélimite.

Q.E.D.

Remarques. 1. On peut se poser la question : d'où vient la différence entre les cas $n=8 l+2$ et $n=8 l+6$ ? A part la raison "algébrique" (l'inexistence d'un spineur parallèle dans le noyau de la forme de Kähler de $C M$ pour $n=8 l+2)$, il y a une raison "topologique" aussi : pour $n=8 l+2$, le seul espace de twisteurs qui est en même temps une variété spinorielle, est $\mathbf{C P}^{4 l+1}$, comme espace de twisteurs de $\mathbf{H P}^{2 l}$. On peut faire l'analogie avec le cas de la dimension complexe paire : ici l'inégalité de Kirchberg peut être améliorée par rapport à la dimension complexe impaire à cause d'une contrainte "algébrique" (sur les valeurs propres de $\Omega$ ), mais une "raison topologique" est le fait que les espaces projectifs ne sont pas spinoriels en dimension complexe paire.

2. Si $\operatorname{Hol}(C M)=0$, on a $U M=S^{n+1}$ (cf. [Bä]) et un raisonnement simple montre que $M=\mathrm{CP}^{m}$. Du fait que $\operatorname{dim}\left(\Sigma_{\frac{m+1}{2}}(M)\right)=C_{m+1}^{\frac{m+1}{2}}$, on trouve $C_{m+1}^{\frac{m+1}{2}}$ spineurs propres de $D$ sur $\mathrm{CP}^{m}$ avec la valeur propre $m+1$.

3. Si $n=8 l-2$, et $\operatorname{Hol}(C M)=\operatorname{Sp}(2 l)$, on trouve un seul spineur parallèle dans $\Sigma_{2 l} C M$, donc un seul spineur propre de $D$ sur $M$ avec la valeur propre $m+1$, dont le conjugué est un spineur propre avec la valeur propre $-(m+1)$.

Exemples. Les seuls exemples connus de variétés quaternioniennes à courbure scalaire positive sont des espaces symétriques. On a trois familles

$$
\mathbf{H P}^{k}=\frac{S p(k+1)}{S p(k) \times S p(1)}, \quad X^{k}=\frac{S U(k+2)}{S(U(k) \times U(2))}, \quad Y^{k}=\frac{S O(k+4)}{S(O(k) \times O(4))},
$$


et 5 exemples correspondant aux groupes exceptionnels. La première famille a comme famille de variétés-limites $M^{4 k+2}=\mathrm{CP}^{2 k+1}$. La famille $X^{k}$ induit comme variétés-limites la famille de variétés des drapeaux ( $k$ impair)

$$
F_{k+2}(2,1)=\left\{\left(V_{1}, V_{2}\right) \mid 0 \in V_{1} \subset V_{2} \subset \mathbf{C}^{k+2}, \operatorname{dim}_{\mathbf{C}} V_{i}=i\right\}
$$

La famille $Y^{k}$ induit comme variétés-limites les espaces homogènes ( $k$ impair)

$$
Z^{4 k+2}=\frac{S O(k+4)}{S(O(k) \times O(3) \times O(2))} .
$$

Pour des détails, on propose [Sa].

Remerciements. Je tiens à remercier Jean Pierre Bourguignon pour les nombreuses idées partagées au cours de nos discussions.

\section{Références}

[Bä] C. BÄR, Real Killing spinors and holonomy, Commun. Math. Phys. 154 (1993), 509-521.

[BGM] C.P.BOYER, K.GALICKI, B.M. MANN, Quaternionic Reduction and Einstein Manifolds, Commun. Anal. Geom. 2 (1993), 229-279.

[Fr] Th. FRIEDRICH, Der erste Eigenwert des Dirac Operators einer kompakten Riemannschen Mannigfaltigkeit nichtnegativer Skalarkrümmung, Math. Nachr. 97 (1980), 117-146.

[He] R. HERMANN, On the Differential Geometry of foliations, Ann. Math. 72 (1960), 445-457.

[Hi1] O. HIJAZI, Opérateurs de Dirac sur les variétés riemanniennes : Minoration des valeurs propres, Thèse de 3ème Cycle, Ecole Polytechnique (1984).

[Hi2] O. HIJAZI, Eigenvalues of the Dirac operator on compact Kähler manifolds, Commun. Math. Phys. 160 (1994), 563-579.

[Ki1] K.-D. KIRCHBERG, An estimation for the first eigenvalue of the Dirac operator on closed Kähler manifolds of positive scalar curvature, Ann. Global Anal. Geom. 3 (1986), 291-325.

[Ki2] K.-D. KIRCHBERG, Compact six-dimensional Kähler spin manifolds of positive scalar curvature with the smallest possible first eigenvalue of the Dirac operator, Math. Ann. 282 (1988), 157-176. 
[Ko] S. KOBAYASHI, On compact Kähler manifolds with positive definite Ricci tensor, Ann. of Math. 74 (1961), 570-574.

[Li] A. LICHNEROWICZ, La première valeur propre de l'opérateur de Dirac pour une variété kählérienne et son cas limite, C. R. Acad. Sci. Paris, t. 311, Série I (1990), 717-722.

[M-S] J.W. MILNOR, J.D. STASHEFF, Characteristic classes, Princeton Univ. Press, New Jersey, 1974.

[O'N1] B. O'NEILL, The fundamental equations of a submersion, Mich. Math J. 13 (1966) 459-469.

[O'N2] B. O'NEILL, Semi-Riemannian geometry, Acad. Press, New York, 1983.

[Sa] S. SALAMON, Quaternionic Kähler Manifolds, Invent. Math. 67 (1982), 143-171.

(A. Moroianu) Centre de Mathématiques, Ecole Polytechnique, Palaiseau 